\title{
Safe and easy hair transplantation utilizing KD spreader
}

\author{
Kuldeep Saxena \\ Cosmazone Fue Hair Transplant Center, Malad West, Mumbai 400064, India.
}

Correspondence to: Dr. Kuldeep Saxena, Cosmazone Fue Hair Transplant Center, 413 Neo Corporate Plaza, Ram Chandra Lane Kanchpada, Malad West, Mumbai 400064, India. E-mail: saxenadrkuldeep@gmail.com

How to cite this article: Saxena K. Safe and easy hair transplantation utilising KD spreader. Plast Aesthet Res 2018;5:5.

http://dx.doi.org/10.20517/2347-9264.2017.67

Received: 11 Sep 2017 First Decision: 2 Feb 2018 Revised: 6 Feb 2018 Accepted: 9 Feb 2018 Published: 27 Feb 2018

Science Editor: Raúl González-García Copy Editor: Jun-Yao Li Production Editor: Huan-Liang Wu

\begin{abstract}
Aim: An atraumatic insertion of graft is key to successful outcomes in this procedure but there is a definite learning curve in available techniques. Many physicians do not continue hair restoration practice because of the repeated initial failures. The goal of any technique or instrument in follicular unit extraction (FUE) is to extract an individual follicular unit without transaction and implanting it in recipient area without producing physical trauma to the follicular unit. This article describes a novel technique in which an innovative instrument, the KD spreader, that addresses all the problems faced by novice physicians during FUE hair transplantation. More importantly unique design of the instrument also solves the problem of fatigue of the operator's hands while performing the hair transplantation procedure.
\end{abstract}

Methods: In this technique, the KD spreader, comprised of a shaft and a hook connected to the shaft are configured to allow the user to work efficiently. Two finger grip of the device provides efficient gripping for fatigue free operation. While performing FUE, the KD spreader provides sufficient traction at the time of scoring. During implantation of the grafts in premade coronal slits, this device provides adequate dilation and maximum visualization of the slit. Attachment of the graft holding plate reduces the chances of dehydration of the graft.

Results: In this study, we have observed use of KD spreader definitely showed advantages over the conventional technique forceps. The KD spreader provides efficient gripping, maximum visualization of slit and better stretching force for the dilatation of the slit to adjust even bigger size follicular unit grafts.

Conclusion: The KD spreader may improve ability of the beginners to perform FUE extraction and implantation smoothly.

Keywords: KD spreader, follicular unit extraction hair transplant, coronal slits graft implantation, graft extraction, hydration of graft

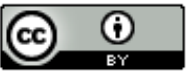

(C) The Author(s) 2018. Open Access This article is licensed under a Creative Commons Attribution 4.0 International License (https://creativecommons.org/licenses/by/4.0/), which permits unrestricted use, sharing, adaptation, distribution and reproduction in any medium or format, for any purpose, even commercially, as long as you give appropriate credit to the original author(s) and the source, provide a link to the Creative Commons license, and indicate if changes were made. 


\section{INTRODUCTION}

Follicular unit extraction (FUE) is a minimally invasive hair transplantation procedure, where each hair follicular unit is individually harvested from the donor area on the scalp or from other parts of the body and then implanted directly into the recipient area. FUE hair transplant surgery has gained popularity in the last few years among patients due to its simplicity and ability to produce less visible scarring, allowing the patients to wear their hair short. The technique of the FUE procedure not only attracts patients who have fear of knives, stitches and linear scarring over the donor site but also physicians who wish to start hair restoration procedures at their clinics. With the evolution of the FUE method, this hair restoration surgery is gaining popularity all over the world.

After working in the field of hair transplantation for more than a decade, the authors have realized there is a definite learning curve in this field of medicine. Many physicians do not continue hair restoration practice because of the repeated initial failures.

Repeated improvement in technique and instrumentation may improve ability of beginners to perform FUE extraction and implantation smoothly. The goal of any technique or instrument in FUE is to extract an individual follicular unit without transection and implant it in the recipient site without producing physical trauma to the follicular unit. As hair transplant is a time-consuming procedure, your technique and instruments should also give you opportunity to work without fatigue and exhaustion. To fulfill these goals, this author created an instrument which can address the existing limitations of the FUE hair restoration procedure.

\section{EXISTING INSTRUMENTS AND ITS LIMITATIONS}

- Manual stretching does not provide sufficient traction for scoring.

- Manual traction can cause operator fatigue especially during procedures which can last for several hours.

- Tough scalp requires extra stretching of tissue that cannot be achieved by available methods.

- Conventional skin hooks do not provide efficient gripping for desired stretching force.

- FUE over lower occipital region require maximum scalp stretch near the FUE site that is not possible by current available methods.

- While performing body hair transplant, skin is extremely pliable and tensioners or spreaders are needed to stabilized flexibility of the tissue.

Looking at these hurdles, the authors desired to develop a user - friendly instrument that can provide donor tension or traction without producing operator fatigue, specifically their hands.

I have developed a unique instrument - the KD' spreader - in response to this problem [Figure 1] ${ }^{[1]}$. This device is comprised of a shaft and a hook connected to the shaft that are configured to allow the user to work efficiently. The device uses two finger grips which are positioned on the surface of the shaft for providing efficient grip so that the desired stretching force may be applied without much fatigue to the operator muscles. This device is extremely useful while FUE is performed on tough scalp where manual stretching does not provide sufficient traction for scoring.

While performing FUE extraction, we start with a circular incision with average rotational force in which our power machine is set. We adjust rotational force as per our requirement. When scalp is tough we have to increase rotational force while when the scalp is soft, we have to decrease rotational force. When we increase rotational force to initiate circular incision on tight scalp, an opposing friction or resistance force is generated. If the applied force exceeds the friction force, chances of transection of grafts greatly increases. KD spreader provides desired stretching exactly at the site of FUE [Figure 2] ${ }^{[2]}$. 

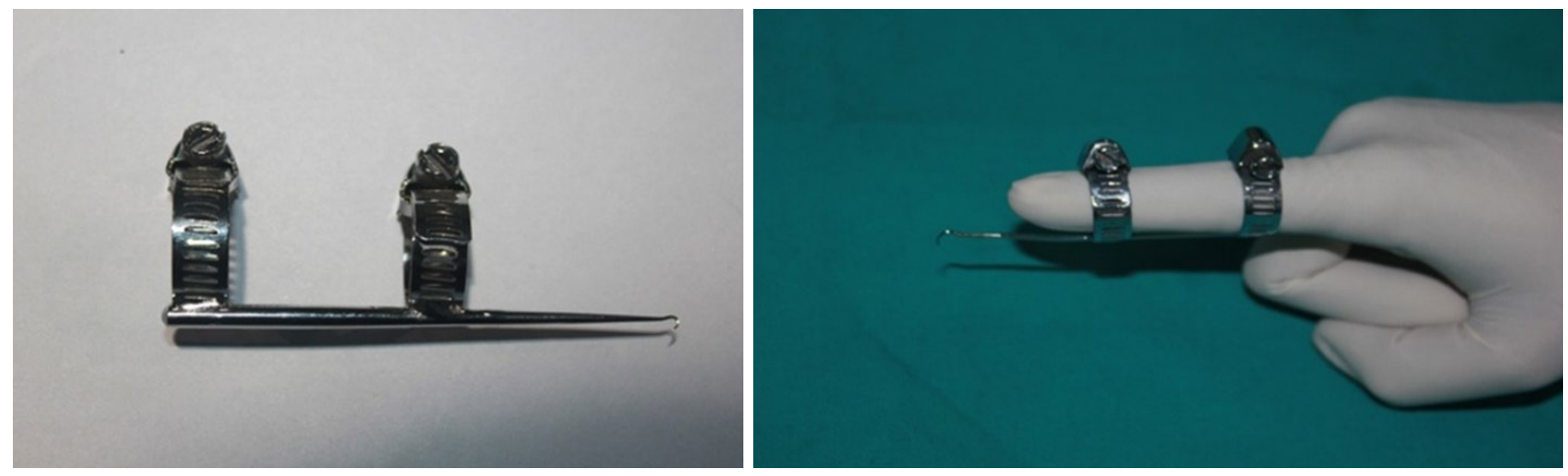

Figure 1. The KD spreader, a modified skin spreader

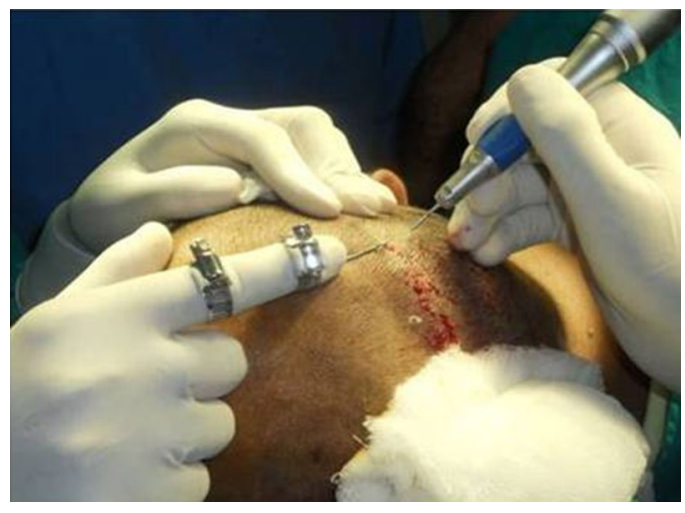

Figure 2. Stretching with tensioner above the extraction site

During graft harvesting, the KD spreader provides the following advantages:

- It fits the index finger of the non-dominant hand of the user.

- Terminal portion of the device gently lifts and stretches the donor area just above the extraction site.

- Desired stretch can be achieved by pulling force at the time of scoring.

- While wearing the device, the assistant's hand is free to hold other objects without keeping the device on the surface, thus reducing the procedure time.

\section{HASSLE FREE SMOOTH EXTRACTION}

Lateral or upward traction is used for removal of the grafts after it has been punched.

This often is the most difficult part, particularly in a tight scalp or if the unit has hairs with divergent angles of emergence. Upward and lateral manual traction or pressure just near the FUE site can smoothen extraction, and the graft may be pulled out easily with forceps in one hand. The author questions when you have to extract 1500 or 2000 grafts with this manual technique, if there increases the likelihood of fatigue to the operator's hand muscles.

Secondly, desired traction cannot be achieved by using manual stretching force. While I was exploring my technique of extraction by using KD spreader, I found that use of KD spreader above the FUE site releases the grafts smoothly, thereby increasing the speed and reducing the chances of decapping of grafts. More importantly, this author has not personally experienced fatigue or pain in his fingers or hand. Even during extraction in tight scalp, we have also found that this technique works exceptionally well [Figure 3]. 

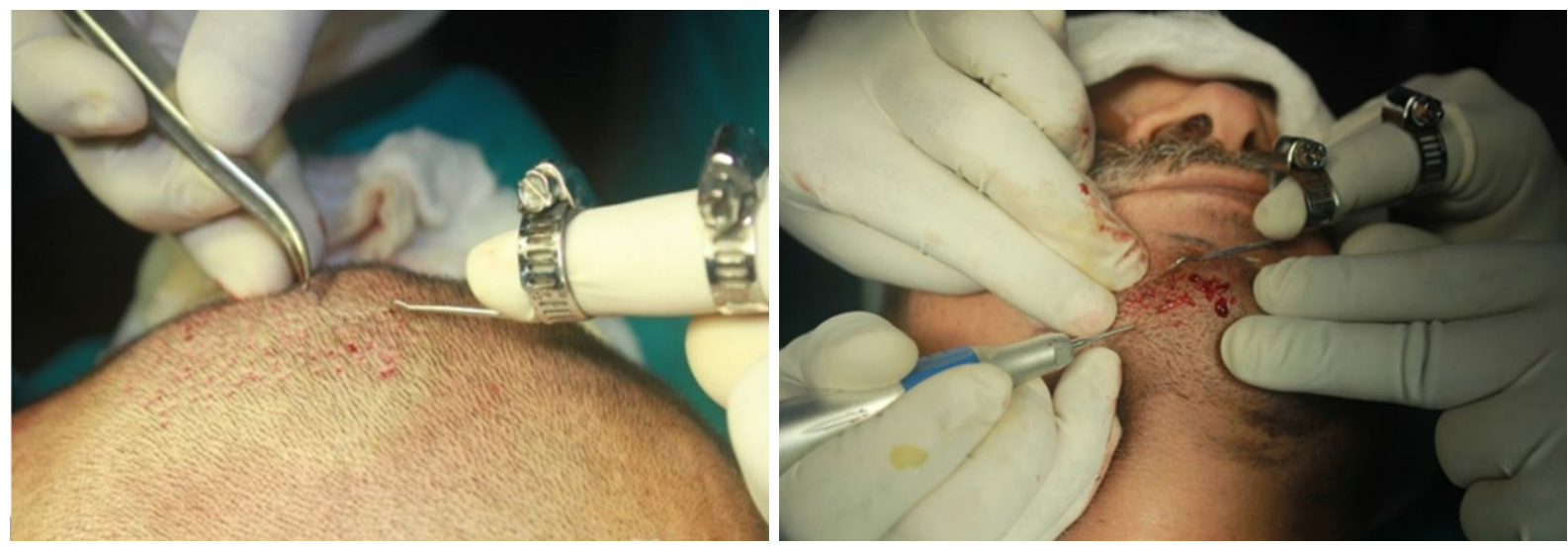

Figure 3. Skin stretching during removal of graft (left) and during beard follicular unit extraction (right)
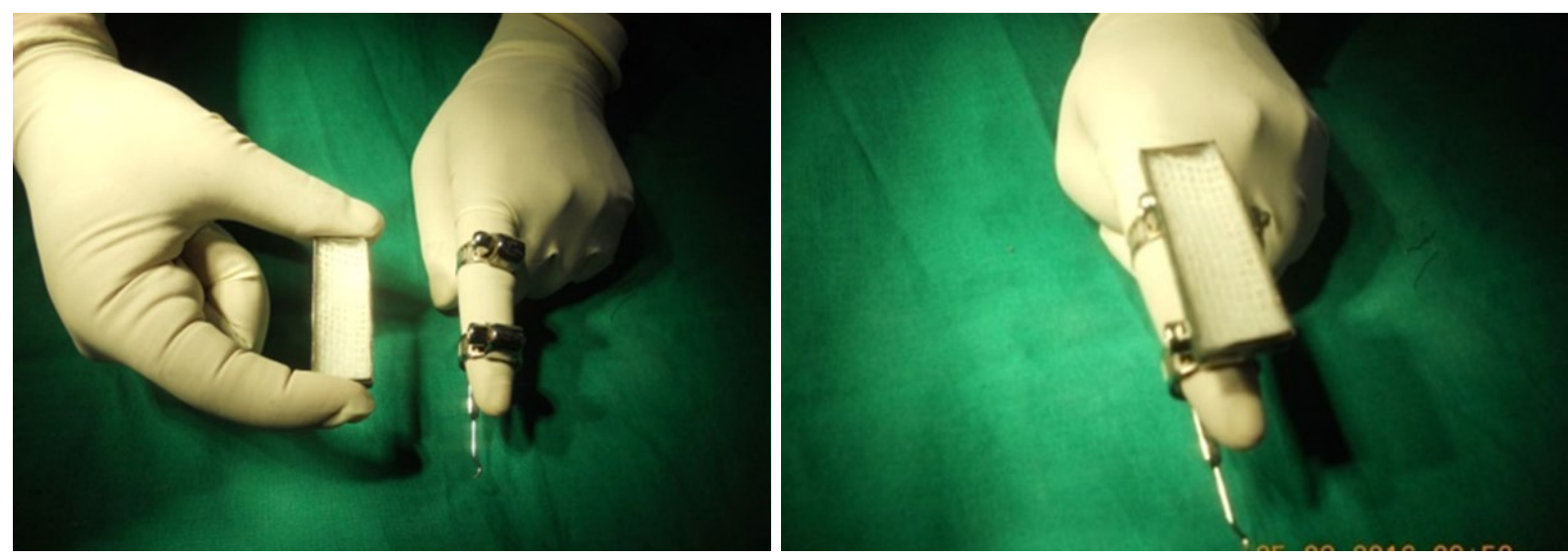

Figure 4. The graft holding plate with KD spreader uses magnets for easy attachment

\section{NOVICE-FRIENDLY IMPLANTATION OF FUE GRAFTS}

Past research and my personal experience suggests that implantation in coronal slits gives more coverage than sagittal slits but there is a long learning curve in this technique when we use forceps ${ }^{[3,4]}$.

Unlike follicular unit transplant graft, where extra fat is obtained for insulation, FUE grafts require extra care during implantation. Several attempts for inserting the graft into a small hole can permanently damage the graft. Experienced assistants and hair transplant experts can perform implantation without any problem but novice operators struggle during implantation.

I have been an advocate of properly hydrated and atraumatic insertion of FUE grafts. While exploring why some assistants or doctors struggle with the learning curve of implantation, I observed several important hurdles that beginners face, which include the following:

- Dilatation of the premade slit and visualization of hole is insufficient to insert graft.

- Gripping the forceps for dilating the premade slit was not comfortable because non-dominant hand grip was not efficient.

- Graft manipulation during graft insertion reduces their confidence.

- There is greater risk of graft dehydration due to prolonged time out of the hydrating solution for beginners compared to trained experts.

- There is an overcrowding of hands when more than one person is performing implantation simultaneously. 

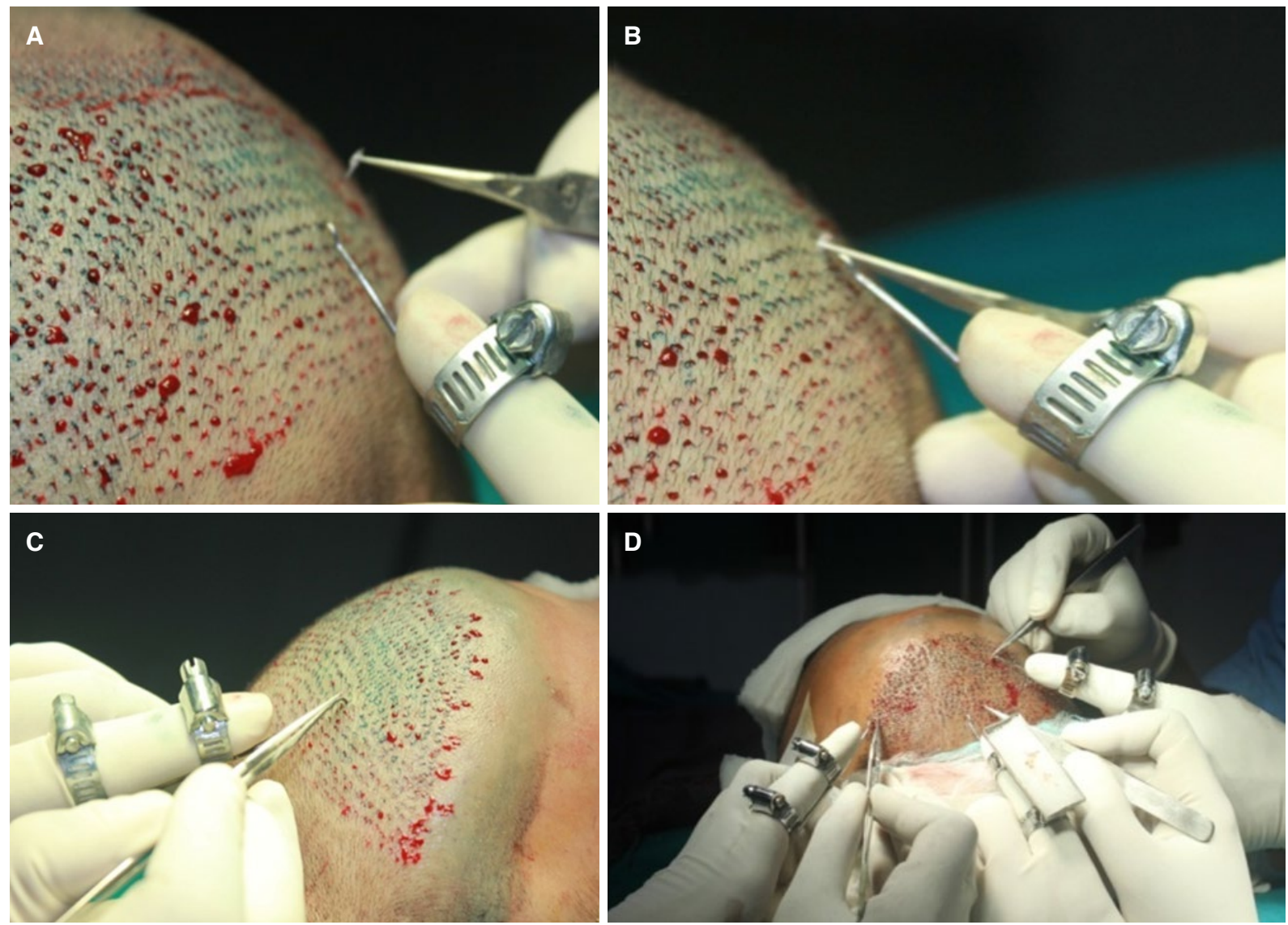

Figure 5. (A) Terminal portion skin hook gently lifts and spreads the most superficial part of skin while another hand grasps the graft using forceps; (B) graft gently slides inside slit while slit is elevated and dilated by the KD spreader; (C) the KD spreader is released and the graft positioned in place; (D) three people performing implantation without overcrowding

After discovering these issues, I concluded that to clear the learning curve, beginners would benefit from a user-friendly device that they would provide maximum visualization ${ }^{[5]}$. In addition, grafts need to be kept in hydrating solution as much as possible and during implantation there should not be overcrowding of hands.

I also noticed that fine motor skills for gentle insertion of the graft develop with time and many beginners are discouraged after failure during initial attempts.

The KD spreader gives beginners the opportunity to exert tissue spreading force as per tissue requirement. It enables them to dilate the slit and get maximum visualization before graft implantation. Since the KD spreader is worn on the index finger, it also avoids overcrowding of the hands in the transplantation site when working simultaneously with another implanter ${ }^{[6]}$.

The upper surface of the KD spreader has a shielded magnet that allows for attachment and detachment of the plates [Figure 4]. This device has another unique feature: at the time of implantation, it provides an opportunity to pick follicular units of your choice as grafts immersed in graft holding solution and placed in a row. This also increases the speed and precision of repetitive graft transfer and placement because grafts and incisions are in the same field of view.

During implantation, the KD spreader works as follows [Figure 5]:

- The implanting operator, who wears the device on the index finger of the non-dominant hand, can adjust the grip. 
- The recipient area is prepared and coronal slits are made.

- The terminal portion of the device gently lifts and spreads the most superficial part of the slit.

- Another hand grasps the graft using forceps.

- The graft gently slides inside the slit while the slit is adequately dilated by the spreader.

- The spreader releases and the graft is positioned in place, thus allowing as many as 3-4 implanters to work together in an organized manner.

\section{ADVANTAGE OF THE KD SPREADER}

There are many advantages to the KD spreader:

- There is lower risk of physical trauma to the graft even in a beginner's hands.

- It improves the ability of beginners to perform FUE.

- It can be used to train inexperienced assistants without jeopardizing quality control.

- It allows 3 or 4 implanters to work in the same field, so a procedure finishes faster.

- It is a low-cost instrument that can be used repeatedly.

- The operator has a reduced risk of fatigue.

- Grafts stay immersed in holding solution, thus limiting the chance of dehydration.

\section{CONCLUSION}

After working in the field of hair transplantation for the last 15 years, I have realized there is a definite learning curve for this procedure. Some physicians and assistants may develop skills very fast while other struggles in clearing the steep learning curve of the FUE procedure. We introduce a novel instrument and technique that may help facilitate this procedure.

The KD spreader may improve the ability of novice operators to perform FUE extraction and implantation smoothly. A reduced learning curve may be necessary to train inexperienced assistants without sacrificing the quality of their FUE transplantation.

\section{DECLARATIONS}

\section{Authors' contributions}

Saxena K contributed solely to the paper.

\section{Financial support and sponsorship}

None.

\section{Conflicts of interest}

There are no conflicts of interest.

\section{Patient consent}

Not applicable.

\section{Ethics approval}

Not applicable.

\section{Copyright}

(c) The Author(s) 2018.

\section{REFERENCES}

1. Ginzburg A. Different orientation for the incision. In: Unger WP, Shapiro R, Unger R, Unger M, editors. Hair Transplantation, Fifth Edition. Boca Raton: CRC Press; 2010. p. 402. 
2. Wong J. Why Lateral Slits. In: Pathomvanich D, Imagawa K, editors. Hair Restoration Surgery in Asians. Tokyo/London: Springer; 2010.

3. Saxena K. FUE Technique \& Instrumentation. In: Mysore V, editor. Hair Transplantation.. New Delhi: Jaypee; 2016. p.194.

4. Saxena K. KD spreader: a slit dilating device and a method of dilating a slit. Proceeding of Haircon 2015 - 7 th Annual Conference of AHRS.

5. Saxena K. Field of invention: surgical instrument used in hair transplantation. Proceeding of ACSICON 2016 - 14th National of Conference of ACS.

6. Saxena K. A Slit dilating device in hair transplantation. Presented at the ISHRS 24th World Congress Las Vegas USA; 2016. 